\title{
Direct Method for Variational Problems via Hybrid of Block-Pulse and Chebyshev Functions
}

\author{
MOHSEN RAZZAGHI ${ }^{a, b, *}$ and HAMID-REZA MARZBAN ${ }^{b}$ \\ a Department of Mathematics and Statistics, Mississippi State University, \\ P.O. Box MA, MS 39762, USA; ${ }^{b}$ Department of Mathematics, \\ Amirkabir University, Tehran, Iran
}

(Received 21 June 1999; In final form 3 November 1999)

A direct method for finding the solution of variational problems using a hybrid function is discussed. The hybrid functions which consist of block-pulse functions plus Chebyshev polynomials are introduced. An operational matrix of integration and the integration of the cross product of two hybrid function vectors are presented and are utilized to reduce a variational problem to the solution of an algebraic equation. Illustrative examples are included to demonstrate the validity and applicability of the technique.

Keywords: Variational problems; Direct method; Hybrid; Chebyshev; Block-pulse

\section{INTRODUCTION}

Orthogonal functions and polynomial series have received considerable attention in dealing with various problems of dynamic systems. The main characteristic of this technique is that it reduces these problems to those of solving a system of algebraic equations, thus greatly simplifying the problem and making it computationally plausible. The approach is based on converting the underlying differential equations into integral equations through integration, approximating various signals involved in the equation, by truncated orthogonal series and using the operational matrix of integration $P$, to eliminate the integral operations. The form

\footnotetext{
*Corresponding author. Fax: 601-325-0005. E-mail: razzaghi@math.msstate.edu.
} 
of $P$ depends on the particular choice for the orthogonal functions. Special attention has been given to applications of Walsh functions [1], block-pulse functions [2], Laguerre series [3], Legendre polynomials [4], Chebyshev polynomials [5], Fourier series [6] and Bessel series [7].

The direct method of Ritz and Galerkin in solving variational problems is well known. Chen and Hsiao [1] introduced the Walsh series method to variational problems. Due to the nature of the Walsh functions, the solution obtained were piecewise constant. References [3-5] used Laguerre polynomials, Legendre polynomials and Chebyshev polynomials respectively to derive continuous solution for the first example in [1]. Furthermore, Razzaghi and Razzaghi [6,8] applied the Fourier series and Taylor series respectively to derive continuous solutions to the second example in [1], which is an application to the heat conduction problem. It is shown that to obtain the Taylor series coefficient, an ill-conditioned matrix commonly known as Hilbert matrix is used. Hence the Taylor series is not suitable for the solution of the second example in [1].

In the present paper we introduce a new direct computational method to solve variational problems. The method consists of reducing the variational problem into a set of algebraic equations by first expanding the candidate function as a hybrid function with unknown coefficients. The hybrid functions, which consist of block-pulse functions plus Chebyshev polynomials are introduced. The operational matrix of integration and the integration of the cross product of two hybrid function vectors are given. These matrices are then used to evaluate the coefficients of the hybrid functions in such a way that the necessary conditions for extremization are imposed. Here we will demonstrate the results by considering the illustrative examples discussed in [3] and the second example in [1]. It is shown that while the same results are obtained in the first example, the hybrid series approach produces an exact solution for the heat conduction problem.

\section{PROPERTIES OF HYBRID FUNCTIONS}

\subsection{Hybrid Functions of Block-Pulse and Chebyshev Functions}

Hybrid functions $b(n, m, t), n=1,2, \ldots, N, m=0,1, \ldots, M-1$ have three arguments, $n$ and $m$ are the order for block-pulse functions and 
Chebyshev polynomials respectively and $t$ is the normalized time. They are defined by

$$
b(n, m, t)= \begin{cases}T_{m}\left(\frac{2 N}{t_{f}} t-2 n+1\right), & \text { for } t \in\left[\left(\frac{n-1}{N}\right) t_{f}, \frac{n}{N} t_{f}\right), \\ 0, & \text { otherwise. }\end{cases}
$$

Here, $T_{m}(t)$ are the well-known Chebyshev polynomials of order $m$ which are orthogonal in the interval $[-1,1]$ with respect to the weight function $w(t)=1 / \sqrt{1-t^{2}}$ and satisfy the following recursive formula:

$$
\begin{aligned}
T_{0}(t) & =1, \quad T_{1}(t)=t, \\
T_{m+1}(t) & =2 t T_{m}(t)-T_{m-1}(t), \quad m=1,2,3, \ldots
\end{aligned}
$$

Since $b(n, m, t)$ consists of block-pulse functions and Chebyshev polynomials, which are both complete and orthogonal, the set of hybrid functions is complete orthogonal set.

\subsection{Function Approximation}

A function $f(t)$ defined over $\left[0, t_{f}\right)$ may be expanded as

$$
f(t)=\sum_{n=1}^{\infty} \sum_{m=0}^{\infty} c(n, m) b(n, m, t),
$$

where

$$
c(n, m)=(f(t), b(n, m, t))
$$

in which $(\cdot, \cdot)$ denotes the inner product.

If the infinite series in Eq. (2) is truncated, then (2) can be written as

$$
f(t) \simeq \sum_{n=1}^{N} \sum_{m=0}^{M-1} c(n, m) b(n, m, t)
$$

or

$$
f(t) \simeq C^{\mathrm{T}} B(t),
$$


where

$$
\begin{aligned}
C= & {[c(1,0), \ldots, c(1, M-1)|c(2,0), \ldots, c(2, M-1)| \cdots} \\
& \mid c(N, 0), \ldots, c(N, M-1)]^{\mathrm{T}}, \\
B(t)= & {[b(1,0, t), \ldots, b(1, M-1, t)|b(2,0, t), \ldots, b(2, M-1, t)| \cdots} \\
& \mid b(N, 0, t), \ldots, b(N, M-1, t)]^{\mathrm{T}} .
\end{aligned}
$$

The integration of the vector $B(t)$ defined in Eq. (4) can be approximated by

$$
\int_{0}^{t} B\left(t^{\prime}\right) \mathrm{d} t^{\prime} \simeq P B(t)
$$

where $P$ is the $N M \times N M$ operational matrix for integration and is given by

$$
P=\left(\begin{array}{ccccc}
E & H & H & \ldots & H \\
0 & E & H & \ldots & H \\
0 & 0 & E & \ldots & H \\
\vdots & \vdots & \vdots & \ldots & \vdots \\
0 & 0 & 0 & \ldots & E
\end{array}\right)
$$

In Eq. (6), we have

$$
H=\frac{t_{f}}{N}\left(\begin{array}{ccccc}
1 & 0 & 0 & \ldots & 0 \\
0 & 0 & 0 & \ldots & 0 \\
\frac{-1}{3} & 0 & 0 & \ldots & 0 \\
\vdots & \vdots & \vdots & \ldots & \vdots \\
\frac{(-1)^{M}-1}{2 M(M-2)} & 0 & 0 & \ldots & 0
\end{array}\right)
$$


and $E$ is the operational matrix of integration for Chebyshev polynomials on the interval $\left[((n-1) / N) t_{f},(n / N) t_{f}\right]$ given in [5] by

$E=$

$\frac{t_{f}}{N}\left(\begin{array}{cccccccc}\frac{1}{2} & \frac{1}{2} & 0 & 0 & \ldots & 0 & 0 & 0 \\ \frac{-1}{8} & 0 & \frac{1}{8} & 0 & \ldots & 0 & 0 & 0 \\ \frac{-1}{6} & \frac{-1}{4} & 0 & \frac{1}{12} & \ldots & 0 & 0 & 0 \\ \vdots & \vdots & \vdots & \vdots & \ldots & \vdots & \vdots & \vdots \\ \frac{-1}{2(M-1)(M-3)} & 0 & 0 & 0 & \ldots & \frac{-1}{4(M-3)} & 0 & \frac{1}{4(M-1)} \\ \frac{-1}{2 M(M-2)} & 0 & 0 & 0 & \ldots & 0 & \frac{-1}{4(M-2)} & 0\end{array}\right)$.

The integration of the cross product of two hybrid function vectors is obtained as

$$
D=\int_{0}^{t_{f}} B(t) B^{\mathrm{T}}(t) \mathrm{d} t
$$

where $D$ is a diagonal matrix, given by

$$
D=\left(\begin{array}{cccc}
L & 0 & \ldots & 0 \\
0 & L & \ldots & 0 \\
\vdots & \vdots & \ddots & \vdots \\
0 & 0 & \ldots & L
\end{array}\right)
$$

In Eq. (8) $L$ is $M \times M$ nonsingular symmetric matrix given by

$$
L=\frac{t_{f}}{2 N}\left(L_{1}+L_{2}\right),
$$


where

$$
\begin{aligned}
& L_{1}= \\
& \left(\begin{array}{cccccc}
1 & 0 & \frac{-1}{3} & \cdots & \frac{(-1)^{M-1}-1}{2(M-1)(M-3)} & \frac{(-1)^{M}-1}{2 M(M-2)} \\
0 & \frac{-1}{3} & 0 & \cdots & \frac{(-1)^{M}-1}{2 M(M-2)} & \frac{(-1)^{M+1}-1}{2(M+1)(M-1)} \\
\frac{-1}{3} & 0 & \frac{-1}{15} & \cdots & \frac{(-1)^{M+1}-1}{2(M+1)(M-1)} & \frac{(-1)^{M+2}-1}{2(M+2) M} \\
0 & \frac{-1}{15} & 0 & \cdots & \frac{(-1)^{M+2}-1}{2(M+2) M} & \frac{(-1)^{M+3}-1}{2(M+3)(M+1)} \\
\vdots & \vdots & \vdots & \vdots & \vdots & \vdots \\
\frac{(-1)^{M-1}-1}{2(M-1)(M-3)} & \frac{(-1)^{M}-1}{2 M(M-2)} & \frac{(-1)^{M+1}-1}{2(M+1)(M-1)} & \cdots & \frac{-1}{(2 M-3)(2 M-5)} & 0 \\
\frac{(-1)^{M}-1}{2 M(M-2)} & \frac{(-1)^{M+1}-1}{2(M+1)(M-1)} & \frac{(-1)^{M+2}-1}{2(M+2) M} & \cdots & 0 & \frac{-1}{(2 M-1)(2 M-3)}
\end{array}\right)
\end{aligned}
$$

and

$$
\begin{aligned}
& L_{2}= \\
& \left(\begin{array}{cccccc}
1 & 0 & \frac{-1}{3} & \cdots & \frac{(-1)^{M-1}-1}{2(M-1)(M-3)} & \frac{(-1)^{M}-1}{2 M(M-2)} \\
0 & 1 & 0 & \cdots & \frac{(-1)^{M-2}-1}{2(M-2)(M-4)} & \frac{(-1)^{M-1}-1}{2(M-1)(M-3)} \\
\frac{-1}{3} & 0 & 1 & \cdots & \frac{(-1)^{M-3}-1}{2(M-3)(M-5)} & \frac{(-1)^{M-2}-1}{2(M-2)(M-4)} \\
0 & \frac{-1}{3} & 0 & \cdots & \frac{(-1)^{M-4}-1}{2(M-4)(M-6)} & \frac{(-1)^{M-3}-1}{2(M-3)(M-5)} \\
\vdots & \vdots & \vdots & \vdots & \vdots & \vdots \\
\frac{(-1)^{M-1}-1}{2(M-1)(M-3)} & \frac{(-1)^{M-2}-1}{2(M-2)(M-4)} & \frac{(-1)^{M-3}-1}{2(M-3)(M-5)} & \cdots & 1 & 0 \\
\frac{(-1)^{M}-1}{2 M(M-2)} & \frac{(-1)^{M-1}-1}{2(M-1)(M-3)} & \frac{(-1)^{M-2}-1}{2(M-2)(M-4)} & \cdots & 0 & 1
\end{array}\right)
\end{aligned}
$$

\section{HYBRID FUNCTIONS DIRECT METHOD}

Consider the problem of finding the extremum of the functional

$$
J(x)=\int_{0}^{1} F[t, x(t), \dot{x}(t)] \mathrm{d} t .
$$


The necessary condition for $x(t)$ to extremize $J(x)$ is that it should satisfy the Euler-Lagrange equation

$$
\frac{\partial F}{\partial x}-\frac{\mathrm{d}}{\mathrm{d} t}\left(\frac{\partial F}{\partial \dot{x}}\right)=0
$$

with appropriate boundary conditions. However, the above differential equation can be integrated easily only for simple cases. Thus numerical and direct methods such as the well-known Ritz and Galerkin methods have been developed to solve variational problems. Here we consider a Ritz direct method for solving Eq. (9) using the hybrid functions of Section 2.

Suppose the rate variable $\dot{x}(t)$ can be expressed as

$$
\dot{x}(t)=C^{\mathrm{T}} B(t) .
$$

Using Eq. (5), $x(t)$ can be represented as

$$
\begin{aligned}
x(t) & =\int_{0}^{t} \dot{x}\left(t^{\prime}\right) \mathrm{d} t^{\prime}+x(0) \\
& =C^{\mathrm{T}} P B(t)+[x(0), 0, \ldots, 0|x(0), 0, \ldots, 0| \cdots \mid x(0), 0, \ldots, 0]^{\mathrm{T}} B(t) .
\end{aligned}
$$

We can also express $t$ in terms of $B(t)$ as

$$
\begin{aligned}
t= & {\left[\frac{1}{2 N}, \frac{1}{2 N}, 0, \ldots, 0\left|\frac{3}{2 N}, \frac{1}{2 N}, \ldots, 0, \ldots, 0\right| \ldots\right.} \\
& \left.\mid \frac{2 N-1}{2 N}, \frac{1}{2 N}, 0, \ldots, 0\right]^{\mathrm{T}} B(t)=\mathrm{d}^{\mathrm{T}} B(t) .
\end{aligned}
$$

Substituting Eqs. (10)-(12) in (9) the functional $J(x)$ becomes a function of $c(n, m), n=1,2, \ldots, N, m=0,1,2, \ldots, M-1$. Hence, to find the extremum of $J(x)$ we solve

$$
\frac{\partial J}{\partial c(n, m)}=0 \quad n=1,2, \ldots, N, m=0,1, \ldots, M-1 .
$$

The above procedure is now used to solve the following variational problems. 


\section{ILLUSTRATIVE EXAMPLES}

Example 1 Consider the problem of finding the minimum of the functional [3]

$$
J(x)=\int_{0}^{1}\left[\dot{x}^{2}+t \dot{x}+x^{2}\right] \mathrm{d} t
$$

with boundary conditions

$$
x(0)=0, \quad x(1)=\frac{1}{4} .
$$

Using Eqs. (10)-(12) in (14) we get

$$
J(x)=\int_{0}^{1}\left[C^{\mathrm{T}} B(t) B^{\mathrm{T}}(t) C+C^{\mathrm{T}} B(t) B^{\mathrm{T}}(t) d+C^{\mathrm{T}} P B(t) B^{\mathrm{T}}(t) P^{\mathrm{T}} C\right] \mathrm{d} t
$$

or

$$
J(x)=C^{\mathrm{T}} D C+C^{\mathrm{T}} D d+C^{\mathrm{T}} P D P^{\mathrm{T}} C
$$

where

$$
D=\int_{0}^{1} B(t) B^{\mathrm{T}}(t) \mathrm{d} t
$$

Equation (10) and the boundary conditions in Eq. (15) imply

$$
x(1)=C^{\mathrm{T}} \int_{0}^{1} B(t) \mathrm{d} t=\frac{1}{4} .
$$

Let

$$
\begin{aligned}
v & =\int_{0}^{1} B(t) \mathrm{d} t \\
& =\frac{1}{N}\left[1,0, \frac{-1}{3}, \ldots, \frac{(-1)^{M}-1}{2 M(M-2)}|\ldots| 1,0, \frac{-1}{3}, \ldots, \frac{(-1)^{M}-1}{2 M(M-2)}\right]^{\mathrm{T}},
\end{aligned}
$$


hence we have

$$
C^{\mathrm{T}} v=\frac{1}{4} .
$$

We now minimize Eq. (16) subject to (17) using the Lagrange multiplier technique.

Suppose

$$
\tilde{J}(x)=J(x)+\lambda\left(C^{\mathrm{T}} v-\frac{1}{4}\right)
$$

where $\lambda$ is the Lagrange multiplier. Using Eq. (13), we solve

$$
\frac{\partial \tilde{J}}{\partial C}=0, \quad \frac{\partial \tilde{J}}{\partial \lambda}=0
$$

or

$$
2 D C+D d+2 P D P^{\mathrm{T}} C+\lambda v=0, \quad C^{\mathrm{T}} v=\frac{1}{4} .
$$

By choosing $M=3$ and $N=4$ we obtain

$$
t=\left[\frac{1}{8}, \frac{1}{8}, 0, \frac{3}{8}, \frac{1}{8}, 0, \frac{5}{8}, \frac{1}{8}, 0, \frac{7}{8}, \frac{1}{8}, 0\right]^{\mathrm{T}} B(t)=\mathrm{d}^{\mathrm{T}} B(t)
$$

and

$$
v=\left[\frac{1}{4}, 0, \frac{-1}{12}, \frac{1}{4}, 0, \frac{-1}{12}, \frac{1}{4}, 0, \frac{-1}{12}, \frac{1}{4}, 0, \frac{-1}{12}\right]^{\mathrm{T}} .
$$

Table I gives the approximate values of $x(t)$ using the hybrid approach for $M=3$ and $N=4$, together with the exact solutions.

Example 2 Application to the heat conduction problem Consider the extremization of

$$
J=\int_{0}^{1}\left[\frac{1}{2} \dot{x}^{2}-x g(t)\right] \mathrm{d} t=\int_{0}^{1} F(t, x, \dot{x}) \mathrm{d} t,
$$


TABLE I Estimated and exact values of $x(t)$

\begin{tabular}{lcc}
\hline$t$ & Hybrid & Exact \\
\hline 0.0 & 0.000000 & 0.000000 \\
0.1 & 0.041949 & 0.041950 \\
0.2 & 0.079318 & 0.079317 \\
0.3 & 0.112472 & 0.112473 \\
0.4 & 0.141750 & 0.141750 \\
0.5 & 0.167444 & 0.167442 \\
0.6 & 0.189805 & 0.189806 \\
0.7 & 0.209065 & 0.209065 \\
0.8 & 0.225412 & 0.225413 \\
0.9 & 0.239011 & 0.239012 \\
1 & 0.249999 & 0.250000 \\
\hline
\end{tabular}

where $g(t)$ is a known function satisfying

$$
\int_{0}^{1} g(t) \mathrm{d} t=-1
$$

with the boundary conditions

$$
\dot{x}(0)=0, \quad \dot{x}(1)=0 .
$$

Schechter [9] gave a physical interpretation for this problem by noting an application in heat conduction and [1] considered the case where $g(t)$ is given by

$$
g(t)= \begin{cases}-1, & 0 \leq t<\frac{1}{4}, \quad \frac{1}{2} \leq t<1 \\ 3, & \frac{1}{4} \leq t<\frac{1}{2}\end{cases}
$$

and gave an approximate solution using Walsh functions.

The exact solution is

$$
x(t)= \begin{cases}\frac{1}{2} t^{2}, & 0 \leq t \leq \frac{1}{4} \\ -\frac{3}{2} t^{2}+t-\frac{1}{8}, & \frac{1}{4} \leq t \leq \frac{1}{2} \\ \frac{1}{2} t^{2}-t+\frac{3}{8}, & \frac{1}{4} \leq t \leq 1\end{cases}
$$


Here, we solve the same problem using hybrid functions. First we assume

$$
\dot{x}(t)=C^{\mathrm{T}} B(t) .
$$

In view of Eq. (20), we write (18) as

$$
J=\frac{1}{2} \int_{0}^{1} \dot{x}^{2}(t) \mathrm{d} t+4 \int_{0}^{1 / 4} x(t) \mathrm{d} t-4 \int_{0}^{1 / 2} x(t) \mathrm{d} t+\int_{0}^{1} x(t) \mathrm{d} t
$$

or

$$
\begin{aligned}
J= & \frac{1}{2} \int_{0}^{1} C^{\mathrm{T}} B(t) B^{\mathrm{T}} C \mathrm{~d} t+4 C^{\mathrm{T}} P \int_{0}^{1 / 4} B(t) \mathrm{d} t \\
& -4 C^{\mathrm{T}} P \int_{0}^{1 / 2} B(t) \mathrm{d} t+C^{\mathrm{T}} P \int_{0}^{1} B(t) \mathrm{d} t .
\end{aligned}
$$

Let

$$
v(t)=\int_{0}^{t} B\left(t^{\prime}\right) \mathrm{d} t^{\prime}
$$

then, using Eqs. (7) and (21) we have

$$
J=\frac{1}{2} C^{\mathrm{T}} D C+C^{\mathrm{T}} P\left[4 v\left(\frac{1}{4}\right)-4 v\left(\frac{1}{2}\right)+v(1)\right] .
$$

The boundary conditions in Eq. (19) can be expressed in terms of hybrid functions as

$$
C^{\mathrm{T}} B(0)=0, \quad C^{\mathrm{T}} B(1)=0 .
$$

We now minimize Eq. (22) subject to (23) using the Lagrange multiplier technique. Suppose

$$
J^{*}=J+\lambda_{1} C^{\mathrm{T}} B(0)+\lambda_{2} C^{\mathrm{T}} B(1),
$$

where $\lambda_{1}$ and $\lambda_{2}$ are the two multipliers. Using Eq. (13) we obtain

$$
\frac{\partial J^{*}}{\partial C}=D C+P\left[4 v\left(\frac{1}{4}\right)-4 v\left(\frac{1}{2}\right)+v(1)\right]+\lambda_{1} B(0)+\lambda_{2} B(1)=0 .
$$


By choosing $M=3$ and $N=4$, Eqs. (23) and (24) define a set of 14 simultaneous linear algebraic equations from which the coefficient vector $C$ and the multipliers $\lambda_{1}$ and $\lambda_{2}$ can be found. The vector $C^{\mathrm{T}} P$ is

$$
C^{\mathrm{T}} P=\frac{1}{64}\left[\frac{2}{3}, 1, \frac{1}{3}, 2,-1,-\frac{10}{3},-3, \frac{1}{3},-\frac{22}{3},-1, \frac{1}{3}\right]^{\mathrm{T}} .
$$

Further, to define $x(t)$ for $t$ in the interval $\left[0, \frac{1}{4}\right]$, we map $\left[0, \frac{1}{4}\right]$ into $[-1,1]$ by mapping $t$ into $8 t-1$ and similarly for the other intervals. Using Eq. (25), we get

$$
x(t)=\left\{\begin{array}{l}
\frac{1}{64}\left[\frac{2}{3}+(8 t-1)+\frac{1}{3}\left[\frac{3}{2}(8 t-1)^{2}-\frac{1}{2}\right]\right]=\frac{1}{2} t^{2}, \\
0 \leq t \leq \frac{1}{4} \\
\frac{1}{64}\left[2-(8 t-3)-\left[\frac{3}{2}(8 t-3)^{2}-\frac{1}{2}\right]\right]=-\frac{3}{2} t^{2}+t-\frac{1}{8}, \\
\frac{1}{4} \leq t \leq \frac{1}{2} \\
\frac{1}{64}\left[-\frac{10}{3}-3(8 t-5)+\frac{1}{3}\left[\frac{3}{2}(8 t-5)^{2}-\frac{1}{2}\right]\right]=\frac{1}{2} t^{2}-t+\frac{3}{8}, \\
\frac{1}{2} \leq t \leq \frac{3}{4} \\
\frac{1}{64}\left[-\frac{22}{3}-(8 t-7)+\frac{1}{3}\left[\frac{3}{2}(8 t-7)^{2}-\frac{1}{2}\right]\right]=\frac{1}{2} t^{2}-t+\frac{3}{8}, \\
\frac{3}{4} \leq t \leq 1
\end{array}\right.
$$

which is the exact solution.

\section{CONCLUSION}

The hybrid function operational matrix $P$, together with the integration of the product of two hybrid function vectors $D$, are used to solve the variational problems. In the present method the hybrid functions consist of block-pulse functions plus Chebyshev polynomials and reduce a variational problem into a set of algebraic equations. The matrix $D$ introduced in Eq. (8) is a diagonal matrix, hence making hybrid functions computationally very attractive. It is also shown that the hybrid functions provide an exact solution for the heat conduction problem presented in [1]. 


\section{References}

[1] C.F. Chen and C.H. Hsiao, A Walsh series direct method for solving variational problems, Journal of Franklin Institute, 300 (1975) 265-280.

[2] C. Hwang and Y.P. Shih, Optimal control of delay systems via block-pulse functions, Journal of Optimization Theory and Applications, 45 (1985) 101-112.

[3] C. Hwang and Y.P. Shih, Laguerre series direct method for variational problems, Journal of Optimization Theory and Applications, 39 (1983) 143-149.

[4] R.Y. Chang and M.L. Wang, Shifted Legendre direct method for variational problems, Journal of Optimization Theory and Applications, 39 (1983) 299-307.

[5] I.R. Horng and J.H. Chou, Shifted Chebyshev direct method for solving variational problems, International Journal of Systems Science, 16 (1985) 855-861.

[6] M. Razzaghi and M. Razzaghi, Fourier series direct method for variational problems, International Journal of Control, 48 (1988) 887-895.

[7] P.N. Paraskevopoulos, P. Sklavounos and G.Ch. Georgiou, The operation matrix of integration for Bessel functions. Journal of Franklin Institute, 327 (1990) 329-341.

[8] M. Razzaghi and M. Razzaghi, Instabilities in the solutions of heat conduction problem using Taylor series and alternative approaches, Journal of Franklin Institute, 326 (1989) 215-224.

[9] R.S. Schechter, The Variation Method in Engineering, McGraw-Hill, New York, 1967. 


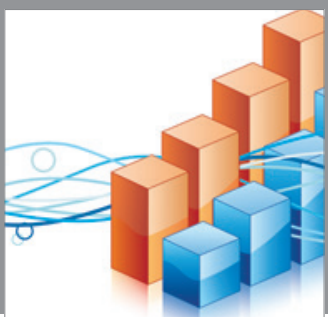

Advances in

Operations Research

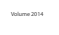

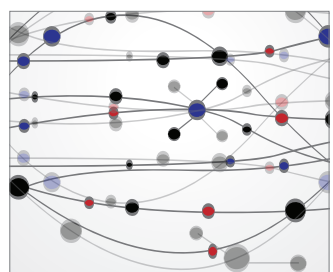

\section{The Scientific} World Journal
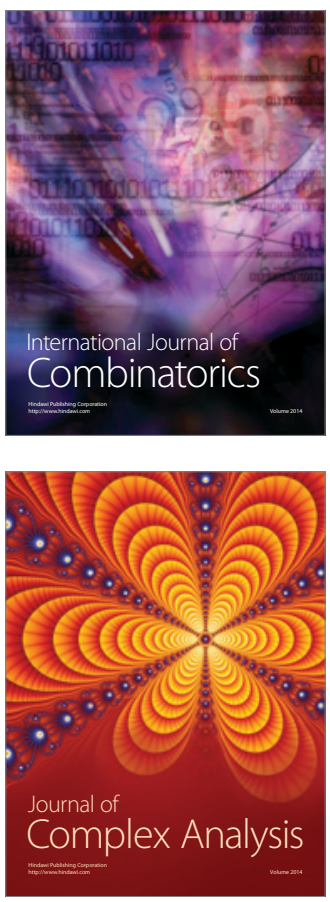

International Journal of

Mathematics and

Mathematical

Sciences
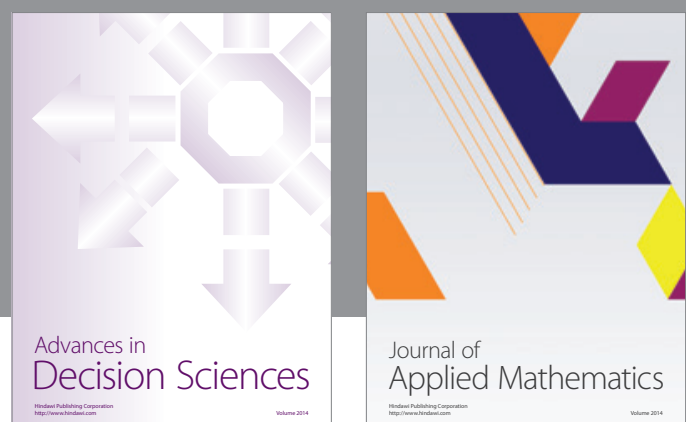

Journal of

Applied Mathematics
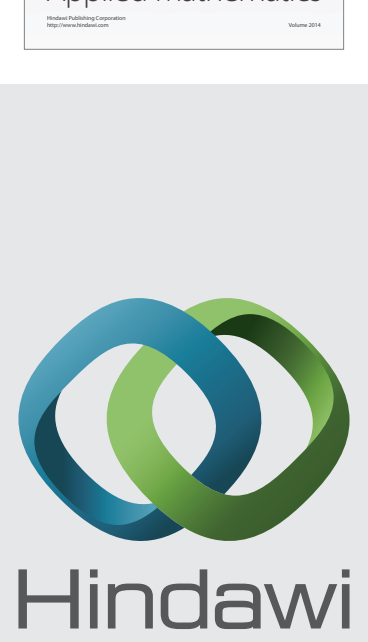

Submit your manuscripts at http://www.hindawi.com
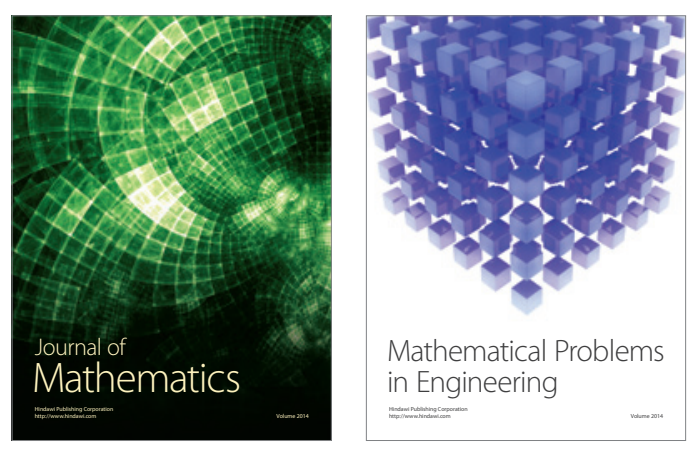

Mathematical Problems in Engineering
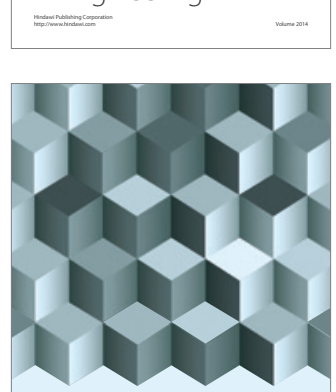

Journal of

Function Spaces
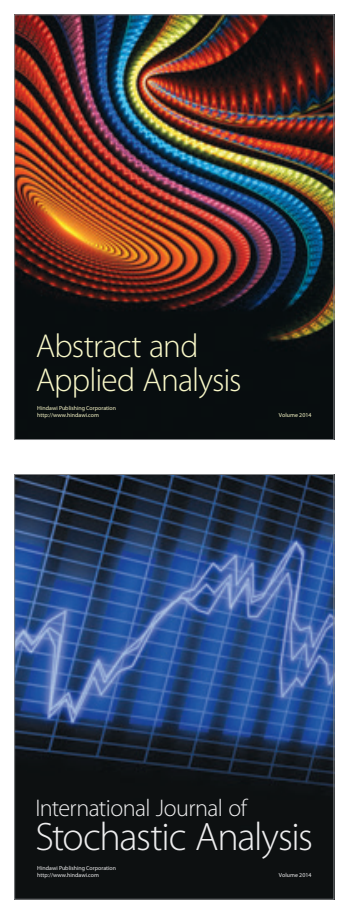

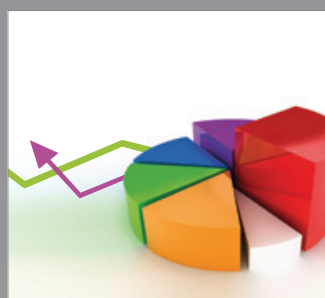

ournal of

Probability and Statistics

Promensencen
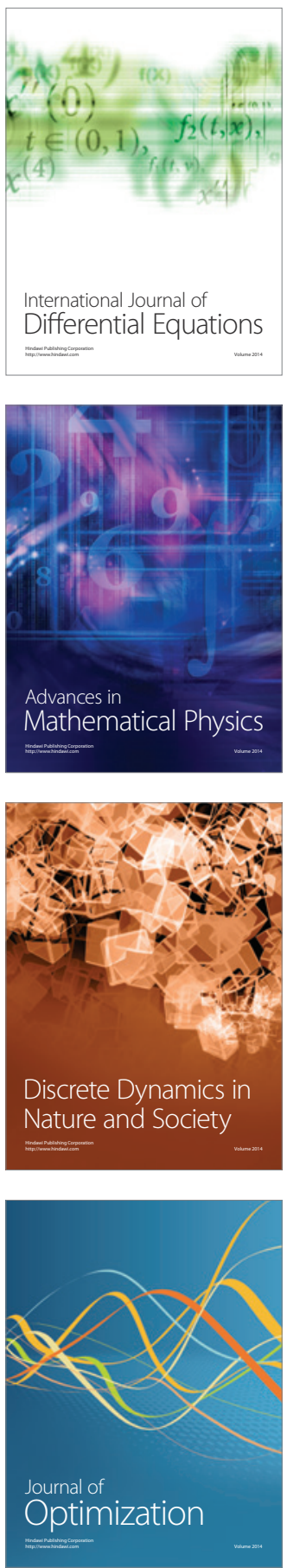